\title{
Documenting the decline of leprosy in Europe: The example of Northern Portugal
}

\author{
GUILHERME GONÇALVES ${ }^{\mathrm{a}}$, ISABEL ANDRADE ${ }^{\mathrm{b}}$, \\ CARLOS PINHEIRO ${ }^{\mathrm{b}}$ \& PAUL E.M. FINE \\ ${ }^{a}$ Unit for Multidisciplinary Research in Biomedicine, Institute of Biomedical \\ Sciences Abel Salazar, University of Porto, Rua de Jorge Viterbo Ferreira \\ 228, 4050-313 Porto, Portugal \\ b ARS Norte, Departamento de Saúde Pública, Rua Anselmo Braancamp \\ 144, 4000-078 Porto, Portugal \\ ${ }^{\mathrm{c}}$ Department of Infectious Disease Epidemiology, London School of Hygiene \\ and Tropical Medicine, Keppel Street, London WC1E 7HT, UK
}

Accepted for publication 5 December 2019

\begin{abstract}
Summary
Background: There is continued uncertainty over trends of leprosy, including in areas with low incidence, where it may be possible to identify areas where $M$ leprae is no longer transmitted or where it no longer causes disease. WHO has reported data on leprosy in the European Region only since 2015.

Methods: Data reported to WHO and published in the Weekly Epidemiological Record were reviewed, Data from five districts in northern Portugal were collected from the National General Directorate of Health and Municipal Health Authorities.

Results: Basic information on 133 leprosy cases has been reported to WHO by thirteen of the 54 states in the European Union since 2015. Data on place of birth of the cases were reported by ten states since 2016 , implying eleven cases possibly attributable to transmission within Europe. Detailed but incomplete data on 38 leprosy cases notified in northern Portugal 1990-2018 are described and discussed. Of those cases which appear to have been autochthonous, none were born after 1966, none were notified after 2007, and the only three notifications after 2005 were for relapses.

Conclusions: Data on leprosy in the European Region are obviously incomplete. The large majority of cases now detected are attributable to infections contracted abroad, but a small number of cases possibly attributable to local transmission are still being identified. Analysis of data from five districts in northern Portugal indicate that this region is no longer endemic for the disease, and that transmission in the area is likely to have ceased at least 40 years ago. The methods illustrated in this paper could be applied to data on leprosy in other regions of Europe, to better define the geographic limits of leprosy today.
\end{abstract}

Keywords: Leprosy, trends, disappearance, Europe, Portugal

Correspondence to: Paul E.M. Fine, London School of Hygiene and Tropical Medicine, London, United Kingdom (e-mail: paul.fine@1shtm.ac.uk)

This work was carried out at the University of Porto in Portugal. 


\section{Introduction}

There has been much discussion of leprosy trends in recent years, stimulated in particular by the 1991 World Health Assembly resolution to "eliminate leprosy as a public health problem" by the year 2000 , defined as a prevalence below 1 per $10,000{ }^{1}$ The latest strategic plan has moved away from an emphasis upon simple prevalence reduction to targets focused upon numbers of newly detected cases with severe (Grade 2) disabilities among children and in total populations, and upon the cessation of legislation which discriminates against leprosy cases and their families. ${ }^{2}$ Trends published in WHO's Weekly Epidemiological Record (WER) indicate that global new case detection is declining slowly, but many questions remain given that several endemic countries fail to report, ${ }^{3-6}$ and the methods for diagnosing, registering and reporting cases have changed over time.

Understandably, there has been most emphasis upon India, Brazil and Indonesia, as these countries account for some $80 \%$ of global leprosy today. Trends in populations with relatively low incidence are also of interest, however, as they may provide evidence of the actual cessation of $M$ leprae transmission (or cessation of the ability of $M$ leprae infection to produce clinical disease). In addition, the presence of only small numbers of patients provides special opportunities for the investigation of risk factors, as one may ask: just why were these individuals among the last apparent cases in this population.

Though leprosy has a long history in Europe, this has received relatively little attention, as it contributes only a very small proportion to the global leprosy burden today. It is widely recognised that the disease was once endemic throughout the region, and that it declined steadily over recent centuries, such that northern Europe has been free of autochthonous disease for several decades. Among the most important events in the history of the disease were the discovery of the leprosy bacillus in Norway in 1873 and the creation of the Norwegian leprosy register in 1856, the first national register of any disease in the world. ${ }^{7}$ This register held data on almost 9,000 patients, up until the last registrations in the 1950s. Several other countries have provided evidence for the cessation of autochthonous disease. Thus the last autochthonous case in the British Isles was identified in 1954, in a young girl whose father had contracted leprosy in Brazil and then immigrated to the UK. ${ }^{8}$ The last case in the Netherlands had onset in $1958 .^{9}$ A recent paper provides evidence that transmission of $M$ leprae ceased within recent decades in the Valencia region of Spain. ${ }^{10}$

The WHO has only published data on leprosy in Europe since 2015. Though the reported data are obviously incomplete, this is an important step in monitoring trends of leprosy in the world. It is appreciated that many of the cases identified now in Europe are in immigrants, or in Europeans who were exposed and infected outside Europe, and are not attributable to autochthonous transmission. The issue of leprosy in migrants has become increasingly important in tracing leprosy trends around the world, and thus WHO has reported data on leprosy in "foreign born" individuals for the years since 2015..$^{3-6}$ This paper discusses some of the problems in tracing the decline of this disease in Europe, with particular reference to the situation in northern Portugal.

\section{Leprosy in Europe today}

Disease reporting systems differ between countries, but leprosy is included on the list of notifiable diseases in virtually all states of the European Region. Table 1 summarises the data as presented in the WER in recent years. According to these numbers, only eighteen "new cases" were reported in 2015, from 5 of the 59 states listed as in the European Region. Of the 
Table 1. Leprosy in the European Region (as reported in the WHO Weekly Epidemiological Record) ${ }^{3-6}$

\begin{tabular}{|c|c|c|c|c|}
\hline Data year & & NCD & Foreign-born & Comments \\
\hline \multirow[t]{12}{*}{2018} & Belgium & 1 & 1 & 54 states \\
\hline & France & 9 & 7 & 14 zero reports \\
\hline & Italy & 5 & 5 & $31 \mathrm{NR}$ (no report) \\
\hline & Netherlands & 2 & 2 & \\
\hline & Portugal & 5 & 3 & 1 NCR with G2D \\
\hline & Russian Fed. & 3 & 0 & \\
\hline & Spain & 7 & 5 & \\
\hline & Sweden & 11 & NR & 39 registered at end 2018 \\
\hline & UK & 7 & NR & \\
\hline & Total & 50 & 23 & \\
\hline & France & 8 & NR & 53 states \\
\hline & Italy & 8 & 6 & 19 zero reports \\
\hline \multirow{9}{*}{2017} & Kazakhstan & 1 & NR & $28 \mathrm{NR}$ (no report) \\
\hline & Netherlands & 3 & 3 & \\
\hline & Portugal & NR & NR & \\
\hline & Spain & 9 & 7 & 1 NCR with G2D \\
\hline & UK & 4 & 4 & \\
\hline & TOTAL & 33 & 20 & 32 registered at end 2017 \\
\hline & Belgium & 3 & NR & 53 states \\
\hline & Germany & 2 & 2 & 24 zero reports \\
\hline & Italy & 12 & 11 & $22 \mathrm{NR}$ \\
\hline \multirow[t]{5}{*}{2016} & Netherlands & 5 & 5 & \\
\hline & Portugal & 4 & 4 & 4 NCR with G2D \\
\hline & UK & 5 & 5 & \\
\hline & Uzbekistan & 1 & NR & 16 registered at end 2016 \\
\hline & TOTAL & 32 & 27 & \\
\hline \multirow[t]{6}{*}{2015} & Germany & 2 & $\begin{array}{l}\text { Not reported for } \\
\text { European countries }\end{array}$ & 59 states \\
\hline & Israel & 2 & & 23 zero reports \\
\hline & Portugal & 2 & & $30 \mathrm{NR}$ \\
\hline & Spain & 8 & & 1 "v" report? \\
\hline & UK & 4 & & \\
\hline & TOTAL & 18 & & \\
\hline 2014 & & & Europe not mentioned & \\
\hline 2013 & & & Europe not mentioned & \\
\hline
\end{tabular}

$\mathrm{NCD}=$ New cases detected; G2D = Grade 2 disability; $\mathrm{NR}=$ no report.

remaining 54 states, 30 provided no report and 23 reported zero new cases (one state is listed as reporting "v" new cases, apparently a typo). ${ }^{3}$ Over the next years 32,33 and then 50 newly detected cases were reported from 53 European states. ${ }^{4-6}$ Data were provided from some of these states on the numbers of cases described as "foreign born" for the years since 2016. ${ }^{4-6}$

Thus over the past four years, a total of 13 European states have reported the detection of at least one "new" leprosy case, the majority (at least 70, of 133 total) being reported in foreignborn individuals. The largest number of new cases has been reported from Italy (12,8 and 5 in the last three years). Only four states have implicitly reported cases in individuals apparently born inside the country: 2 in France, 3 in Italy, 2 in Portugal and 4 in Spain ("implicitly" as these numbers are obtained by subtracting reported foreign born from total new cases detected, though it is possible that place of birth was unknown for some cases). Whether any these cases were autochthonous (attributable to infection within Europe) cannot be inferred from these 
data, as some individuals born in Europe may have become infected elsewhere in the world. Though we may suspect that these data provide far from a complete picture, they are at least consistent with our understanding of the gradual disappearance of leprosy from Europe, with this trend being most advanced in the northern countries of the region.

\section{Leprosy in Portugal}

Statutory notification of infectious diseases was introduced in Portugal in 1901 and leprosy was on the list of reportable diseases from the very beginning. ${ }^{11}$ Specific changes in the procedures to declare, record and process data concerning leprosy cases in Portugal have been described elsewhere. ${ }^{12}$ For most of the time period considered in this study, a notification form (NF) was used for all reportable diseases on the list, including leprosy. The NF was filled out in triplicate, and sent to the Municipal Health Authority (MHA). One copy was kept at the MHA to enable investigations to identify the source of infection and take preventative measures, and one copy was sent to the regional level. The third copy was sent to the General Directorate of Health (GDH) in Lisbon, which provided the basis for national statistics. From 2014 a new surveillance system was implemented, with notifications being made by physicians using an electronic system called SINAVE. ${ }^{13}$

These data have been used to document the decline of leprosy in Portugal from the mid-20th century up until 2016. ${ }^{12,14-16}$ The decline has been precipitous, from more than 100 cases per annum in the 1950s, to approximately 20 per annum in the 1980s to single digits for most years after 1990. These analyses have revealed a shift to older ages and to an increased proportion of multibacillary disease, over time, similar to trends first noted in the Norwegian register. ${ }^{7,12}$ They also revealed that incidence was higher in rural than in urban areas. We here describe data on cases in the five districts of Northern Portugal (NP), from 1990 through 2018, illustrating some of the problems of leprosy surveillance today.

\section{Materials and methods}

This study focuses upon data from five districts in northern Portugal, with a combined population of 3,253,106 in 2011 (Figure 1). Three sources of data were used:

- We consulted official statistics published by the central national GDH. This provided the number of cases by year and district.

- We asked the epidemiological unit of the GDH to provide copies of the paper notification forms (NF). In addition, the NP Regional Health Authority sent an e-mail to all MHAs in $\mathrm{NP}$, asking for the paper notification forms. When available, these forms provided data on: sex, date of birth, place of residence, diagnostic validity (suspect, probable or confirmed), existence of laboratory or other diagnostic data, whether other related cases were known, date when symptoms began, whether the patient was admitted to hospital or died, likely source of infection, occupation, the date on which the physician reported the disease, and date of receipt of the notification by the MHA. The forms changed over time and not all were completely filled.

- The Regional Health Authority also asked the MHAs to provide additional written information (AWI) whenever available, whether from the epidemiological inquiry done after the notification, or other source. Consequent to this request, some of them made additional inquiries on cases, consulting the local computerized database of patients of the Portuguese national health service. Though the AWI was heterogeneous in terms of quantity and quality, it sometimes assisted in distinguishing between newly-detected cases and relapses. It was 


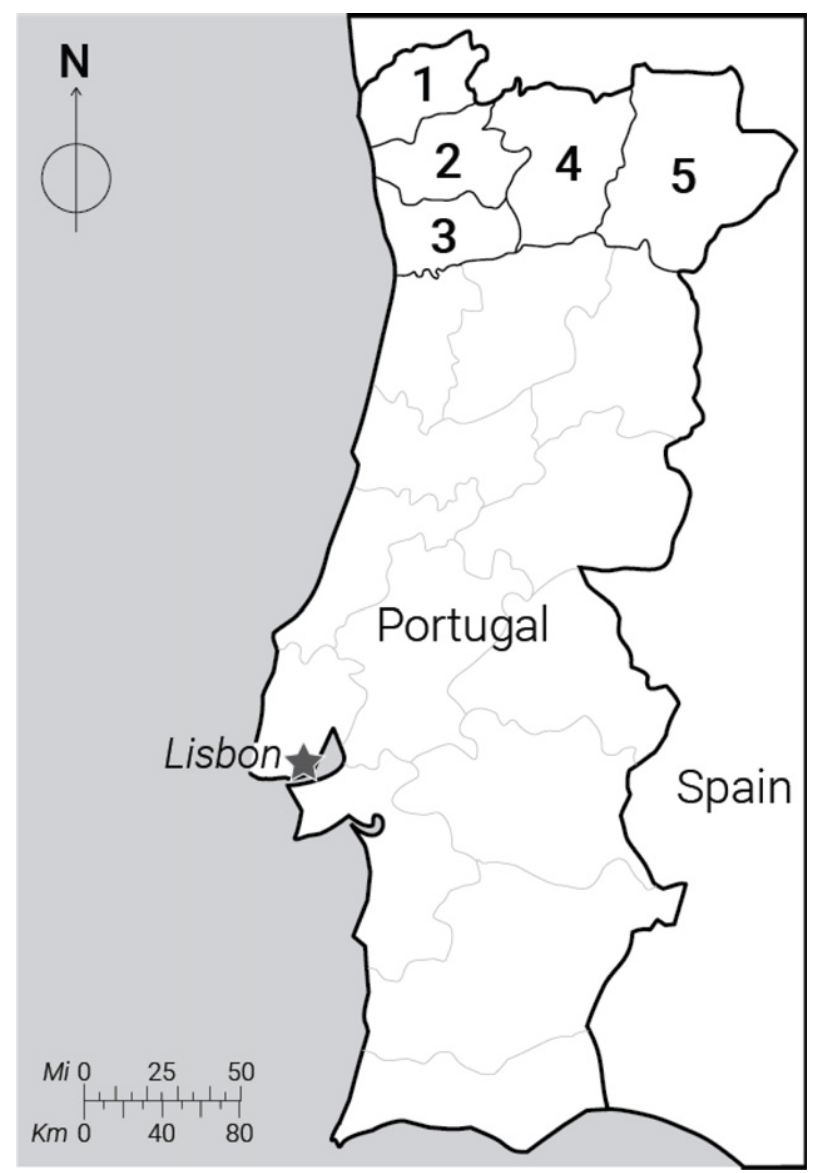

Figure 1. Map of Portugal showing the five northern districts included in this report: $1=$ Viana do Castelo, $2=$ Braga, 3 = Porto, 4 = Vila Real, 5 = Bragança.

also sometimes possible to identify the likely origin of the infection (autochthonous or imported), the clinical classification (Ridley-Jopling and/or WHO pauci/multibacillary), the existence of disabilities, treatment, etc. The AWI was also valuable as a check and to complete information from the notification forms. AWI was available for all cases notified since 2006.

Given the small number of cases, formal statistical analysis is inappropriate, but it is still possible to construct a picture of the epidemiological situation. The study was approved by the Ethics Committee of the Portuguese Northern health region (Comissão de Ética para a Saúde da ARS do Norte) in July 2013.

\section{Results}

Over the years 1990-2013, 154 leprosy cases were reported in the whole country, but only 38 in residents of the five districts of Northern Portugal (NP). Since the year 2014, with the new 


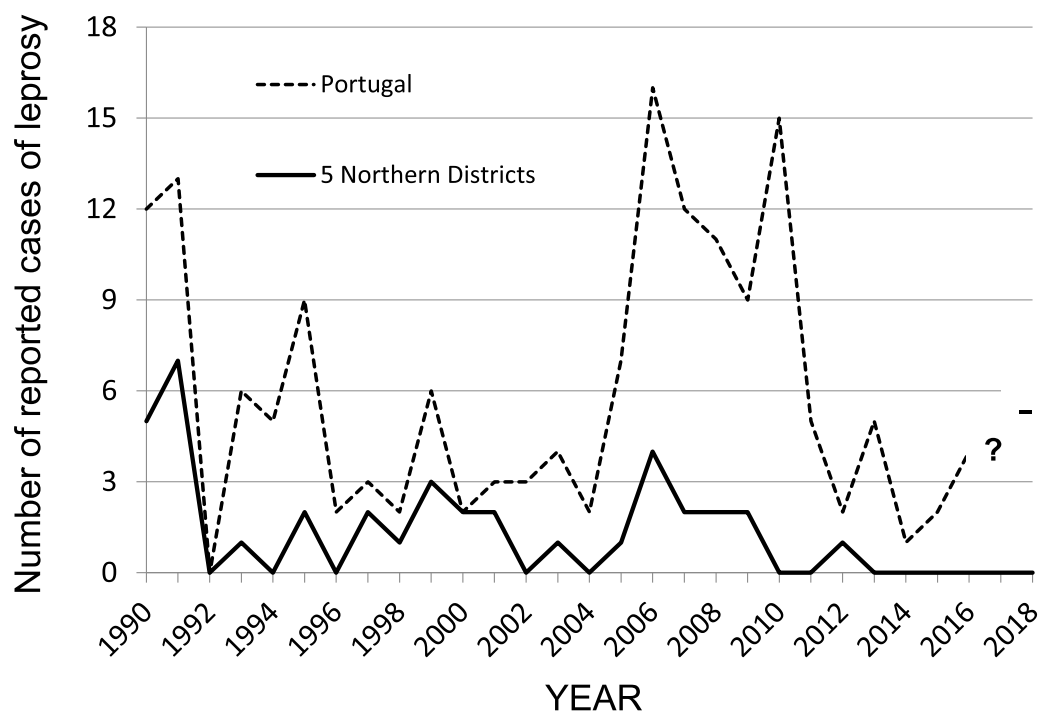

Figure 2. Reported cases of leprosy in Portugal and in the five Northern Portuguese districts, from 1990 to 2018. The number for all Portugal in 2017 is not available.

(SINAVE) reporting system in place, there have been 12 cases reported in Portugal as a whole but none in the five northern districts. ${ }^{6,16}$ (Figure 2).

No written document was available for 13 of the 38 cases published in the official statistics (Table 2). For 10 cases, only the notification form (NF) was available, while for the remaining 15 both the NF and additional written information (AWI) could be consulted. All cases reported after 2005 were in this latter category. The ages of the notified cases with information, 11 females and 14 males, ranged from 15 to 86 years.

For 14 cases there was an explicit clinical classification using the Ridley-Jopling scale (TT to LL) and for another two cases it was described as "multibacilar" (MB) or "paucibacilar" (PB). For 15 of the cases with clinical classification it was clearly mentioned (and sometimes described in detail) that the diagnosis had been lab confirmed. In only one case it was mentioned that the diagnosis was based on clinical information but not yet lab confirmed. Moreover, for three cases with no clinical classification mentioned it was written that diagnosis had been lab confirmed. These classifications are included in Table 2.

It was not always clear from the NF or AWI whether a notification was for newly detected disease, longstanding chronic disease or for relapse, or whether the individual had been notified in the past. Our logic in classifying them was as follows. Of the 15 cases with AWI, 9 had record of onset between 8 and 52 years before the notification date. For 7 of these, there was a record that an earlier treatment course had been successful, and thus we have considered them to be confirmed relapses ("Relapse" in Table 2). There was no information on cure after initial treatment for the other two cases, and the notes mentioned lack of adequate treatment compliance; and thus we have labelled these as probable relapses ("Rel(p)" in Table 2). The remaining 6 cases with AWI have been classified as newly-detected cases ("New" in Table 2), though a doubt may remain for the five cases notified since 2005 among immigrants from Brazil, because of a lack of information from the original country. 
Table 2. Basic characteristics of the 38 leprosy cases reported in North Portugal between 1990 and 2012

\begin{tabular}{|c|c|c|c|c|c|c|c|c|c|c|c|}
\hline \multirow[t]{2}{*}{ Case } & \multicolumn{2}{|c|}{ INFO } & \multirow{2}{*}{$\begin{array}{l}\text { Year } \\
\text { of } \\
\text { Birth }\end{array}$} & \multirow[t]{2}{*}{ Sex } & \multirow{2}{*}{$\begin{array}{l}\text { Year of } \\
\text { Notifi- } \\
\text { cation }\end{array}$} & \multicolumn{2}{|c|}{ ONSET } & \multirow{2}{*}{$\begin{array}{l}\text { Diag* } \\
\text { (conf) }\end{array}$} & \multirow{2}{*}{$\begin{array}{l}\text { New } \\
\text { or } \\
\text { Relapse }\end{array}$} & \multirow{2}{*}{$\begin{array}{l}\text { Autochthonous } \\
\text { or Imported }\end{array}$} & \multirow{2}{*}{$\begin{array}{l}\text { Likely } \\
\text { source } \\
\text { country }\end{array}$} \\
\hline & $\overline{\mathrm{NF}}$ & $\overline{\mathrm{AWI}}$ & & & & $\overline{\mathrm{NF}}$ & AWI & & & & \\
\hline 1 & $\mathrm{Y}$ & $\mathrm{N}$ & 1934 & M & 1990 & 1989 & - & $?(?)$ & New(s) & Autochthonous & \\
\hline 2 & $\mathrm{Y}$ & $\mathrm{N}$ & 1952 & $\mathrm{~F}$ & 1990 & 1986 & - & $?(?)$ & New(s) & Autochthonous & \\
\hline 3 & $\mathrm{Y}$ & $\mathrm{N}$ & 1965 & M & 1990 & NR & - & $?(?)$ & - & Autochthonous & \\
\hline 4 & $\mathrm{~N}$ & $\mathrm{~N}$ & $?$ & $?$ & 1990 & $?$ & $?$ & $?(?)$ & $?$ & $?$ & \\
\hline 5 & $\mathrm{~N}$ & $\mathrm{~N}$ & $?$ & $?$ & 1990 & $?$ & $?$ & $?(?)$ & $?$ & $?$ & \\
\hline 6 & $\mathrm{Y}$ & $\mathrm{N}$ & 1964 & $\mathrm{~F}$ & 1991 & 1990 & - & $?(?)$ & New(s) & Autochthonous & \\
\hline 7 & Y & $\mathrm{N}$ & 1966 & F & 1991 & NR & - & $?(?)$ & - & Autochthonous & \\
\hline 8 & Y & $\mathrm{Y}$ & 1930 & $\mathrm{~F}$ & 1991 & NR & 1971 & LL (L) & $\operatorname{Rel}(p)$ & Autochthonous & \\
\hline 9 & $\mathrm{~N}$ & $\mathrm{~N}$ & $?$ & $?$ & 1991 & $?$ & $?$ & $?(?)$ & $?$ & $?$ & \\
\hline 10 & $\mathrm{~N}$ & $\mathrm{~N}$ & $?$ & $?$ & 1991 & $?$ & $?$ & $?(?)$ & $?$ & $?$ & \\
\hline 11 & $\mathrm{~N}$ & $\mathrm{~N}$ & $?$ & $?$ & 1991 & $?$ & $?$ & $?(?)$ & $?$ & $?$ & \\
\hline 12 & $\mathrm{~N}$ & $\mathrm{~N}$ & $?$ & $?$ & 1991 & $?$ & $?$ & $?(?)$ & $?$ & $?$ & \\
\hline 13 & $\mathrm{Y}$ & $\mathrm{N}$ & 1929 & M & 1993 & 1991 & - & $?(?)$ & New(s) & Autochthonous & \\
\hline 14 & $\mathrm{Y}$ & $\mathrm{N}$ & 1960 & $\mathrm{~F}$ & 1995 & 1993 & - & LL (L) & New(s) & Imported & Africa \\
\hline 15 & $\mathrm{~N}$ & $\mathrm{~N}$ & $?$ & $?$ & 1995 & $?$ & $?$ & $?(?)$ & $?$ & $?$ & \\
\hline 16 & $\mathrm{Y}$ & $\mathrm{Y}$ & 1927 & $\mathrm{~F}$ & 1997 & 1996 & - & LL (L) & New & Autochthonous & \\
\hline 17 & $\mathrm{~N}$ & $\mathrm{~N}$ & $?$ & $?$ & 1997 & $?$ & $?$ & $?(?)$ & $?$ & $?$ & \\
\hline 18 & $\mathrm{Y}$ & $\mathrm{Y}$ & 1932 & M & 1998 & 1990 & 1965 & $\mathrm{BL}(\mathrm{C})$ & Relapse & Autochthonous & \\
\hline 19 & $\mathrm{Y}$ & $\mathrm{N}$ & 1946 & M & 1999 & 1986 & - & $?(\mathrm{~L})$ & $\operatorname{Rel}(\mathrm{s})$ & Imported & Iraq/Egypt \\
\hline 20 & $\mathrm{Y}$ & $\mathrm{N}$ & 1938 & M & 1999 & NR & - & $?(\mathrm{~L})$ & - & Autochthonous & \\
\hline 21 & $\mathrm{~N}$ & $\mathrm{~N}$ & $?$ & $?$ & 1999 & $?$ & $?$ & $?(?)$ & $?$ & $?$ & \\
\hline 22 & $\mathrm{~N}$ & $\mathrm{~N}$ & $?$ & $?$ & 2000 & $?$ & $?$ & $?(?)$ & $?$ & $?$ & \\
\hline 23 & $\mathrm{Y}$ & $\mathrm{Y}$ & 1956 & M & 2000 & 2000 & 1992 & $\mathrm{BL}(\mathrm{L})$ & $\operatorname{Rel}(\mathrm{p})$ & Autochthonous & \\
\hline 24 & $\mathrm{~N}$ & $\mathrm{~N}$ & $?$ & $?$ & 2001 & $?$ & $?$ & $?(?)$ & $?$ & $?$ & \\
\hline 25 & $\mathrm{~N}$ & $\mathrm{~N}$ & $?$ & $?$ & 2001 & $?$ & $?$ & $?(?)$ & $?$ & $?$ & \\
\hline 26 & $\mathrm{~N}$ & $\mathrm{~N}$ & $?$ & $?$ & 2003 & $?$ & $?$ & $?(?)$ & $?$ & $?$ & \\
\hline 27 & $\mathrm{Y}$ & $\mathrm{N}$ & 1925 & M & 2005 & 2002 & - & $?(\mathrm{~L})$ & New(s) & Autochthonous & \\
\hline 28 & $\mathrm{Y}$ & $\mathrm{Y}$ & 1942 & M & 2006 & 2003 & 1970 & LL (L) & Relapse & Autochthonous & \\
\hline 29 & $\mathrm{Y}$ & $\mathrm{Y}$ & 1941 & M & 2006 & 2006 & 1954 & LL (L) & Relapse & Autochthonous & \\
\hline 30 & $\mathrm{Y}$ & $\mathrm{Y}$ & 1981 & M & 2006 & 2006 & 2002 & LL (L) & New & Imported & Brazil \\
\hline 31 & $\mathrm{Y}$ & $\mathrm{Y}$ & 1946 & M & 2006 & 2004 & 1994 & LL (L) & Relapse & Imported & Africa \\
\hline 32 & $\mathrm{Y}$ & $\mathrm{Y}$ & 1982 & $\mathrm{~F}$ & 2007 & 2007 & 2007 & $\mathrm{BB}(\mathrm{L})$ & New & Imported & Brazil \\
\hline 33 & $\mathrm{Y}$ & $\mathrm{Y}$ & 1920 & $\mathrm{~F}$ & 2007 & 2006 & 1974 & LL (L) & Relapse & Autochthonous & \\
\hline 34 & $\mathrm{Y}$ & $\mathrm{Y}$ & 1953 & M & 2008 & 2005 & 1988 & $\mathrm{MB}(\mathrm{L})$ & Relapse & Imported & Venezuela \\
\hline 35 & $\mathrm{Y}$ & $\mathrm{Y}$ & 1979 & M & 2008 & 2005 & 2005 & LL (L) & New & Imported & Brazil \\
\hline 36 & $\mathrm{Y}$ & $\mathrm{Y}$ & 1956 & $\mathrm{~F}$ & 2009 & 2005 & 2006 & LL (L) & New & Imported & Brazil \\
\hline 37 & $\mathrm{Y}$ & $\mathrm{Y}$ & 1994 & $\mathrm{~F}$ & 2009 & 2007 & NR & TT (L) & New & Imported & Brazil \\
\hline 38 & $\mathrm{Y}$ & $\mathrm{Y}$ & 1959 & $\mathrm{~F}$ & 2012 & 2011 & 1988 & PB (L) & Relapse & Imported & Brazil \\
\hline
\end{tabular}

Legend: $\mathrm{NF}=$ notification form; AWI = additional written information from the municipal health authority; NR = not recorded; $(\mathrm{c})=$ confirmed; $(\mathrm{s})=$ suspected; $(\mathrm{p})=$ probable. Diag*for diagnosis: TT-BT-BB-BL-LL (Ridley-Jopling); $\mathrm{MB}=$ multibacilar in case 34 and PB paucibacilar in case 38; Confirmation (conf), with (L) for lab confirmed and (C) for clinical.

Notification forms were available for ten of the cases with no AWI, and seven of these recorded a year of onset. For six of these the interval from recorded onset to notification was between one and four years, and we classify them as suspected new cases ("New(s)" in Table 2). For the seventh (case 19) the interval was 13 years, which we interpreted as a suspected relapse ("Rel(s)" in Table 2). We can make no judgement as to new or relapse status for the 13 cases with no notification form. 
We have classified 15 of the cases as autochthonous and 10 as imported on the basis of the "likely source of infection" information in the notification form (Table 2).

\section{AUTOCHTHONOUS CASES}

Among the 8 autochthonous cases for whom only a NF was available (all reported before 2006), five had records mentioning relatives with leprosy, living in the same household: parents; sisters (in 2 cases); daughter; and an unspecified household relative. The AWI available for the seven other autochthonous cases carried additional information on the individual situations and the likely source of infection, which we summarise here (see Table 2):

- Case 8. A rural woman born in 1930, single and living alone, who had brother and a sister (case 16) with lab-confirmed lepromatous leprosy. The record indicated poor hygiene conditions and lack of compliance with treatment. Original onset in 1971, followed 20 years later by notification for relapse in 1991.

- Case 16. A rural woman born in 1927, who had a sister with confirmed leprosy (case 8). She was notified in 1997, as an LL case having had onset in 1996.

- Case 18. A man born in 1932, who was admitted in 1958 to the only Portuguese hospital specialised in the treatment of leprosy (Hospital Rovisco Pais $=$ HRP). The 1998 notification was considered a relapse (BL form). Other treatments were recorded in 1965, 1990 and 1998.

- Case 23. A rural shepherd born in 1956. His parents had leprosy. His onset was recorded as 1992 in AWI and as 2000 on the notification form in 2000 when he was diagnosed as a (BL) probable relapse.

- Case 28. A man born in 1942. His father had leprosy, was treated and cured, and had been free of symptoms for years. He and the family denied the existence of the disease. In 1970 he had been admitted to HRP and discharged in 1972. He was diagnosed and notified with a relapse (lab-confirmed lepromatous) in 2003 and was treated successfully.

- Case 29. A man born in 1941. His father and grandfather had had leprosy. He was apparently treated successfully after initial diagnosis in 1954, but had relapses in 1985 and 2006.

- Case 33. A rural woman born in 1920. Her husband had had leprosy (we have no details on the case). She was first diagnosed and treated in 1974, but was later diagnosed and treated as a relapse (lab-confirmed lepromatous) in 2006.

\section{IMPORTED CASES}

Of the ten apparently imported cases, six had been born in Brazil, all of whom were reported after 2005. The remaining 4 cases occurred in people born in Portugal who had a history of residence in leprosy endemic areas outside Portugal. Epidemiological interpretation is made difficult by the fact that dates of immigration or of residence abroad were not well or consistently recorded. Of particular interest are cases 36 and 37. Case 36 was a Brazilian woman, born in 1956, who came to Portugal as a teenager but returned to Brazil to study at university, but then later moved back to Portugal, around the year 2000, with her young daughter. Her first symptoms appeared in 2005-2006. She was diagnosed and treated for LL disease in 2009 and rendered bacillary negative but with neurological sequelae. Her daughter, born in Brasil in 1994, was case 37. The daughter was diagnosed in 2009 by biopsy, after clinicians realized her mother's diagnosis, and then successfully treated for tuberculoid disease. Given this history, we suspect the girl was infected, probably via her mother, before leaving Brazil (though it is possible that the infection event occurred after the mother and 
daughter arrived in Portugal). This girl was the youngest by far of all the 38 cases identified in northern Portugal since 1990.

Visible deformities, consistent with WHO Grade 2 disability, were reported in 3 newly detected cases and 7 relapses. Four cases were described in the AWI as experiencing strong social stigma, which impeded contact with health professionals: one of them had refused an admission to hospital in 1995 and another was described as living in "very poor hygiene conditions and poor compliance with medication".

Sixteen cases lived in rural, 4 in suburban and 5 in urban areas. Occupation was recorded for 21 cases: rural workers (6), house wives (5), unemployed (3) and retired (2) were the most frequent occupations mentioned.

\section{Discussion}

These data illustrate several problems in monitoring and understanding leprosy in Europe today. Leprosy is a rare disease, of relatively low priority to health services in Europe, and attracts little attention except among the small number of specialists and practitioners who see cases. What data there are come from a variety of sources, are not always consistent, and are likely to be incomplete. Few physicians in Europe are familiar with the disease, and so the diagnosis is likely to be missed and delayed. The policies for notifying relapses may not be clear within or consistent between countries. Though "onset" year is sometimes recorded, it is widely recognised that this is often unknown or unreliable. Given the stigma associated with the disease, there are likely to be confidentiality restrictions in some countries which further complicate data collection, availability and analysis.

There are special problems associated with the reporting and interpretation of "foreign born" cases, insofar as some may reflect recent onsets, newly detected in the reporting country, others may have been detected before (and even reported to WHO as "new") in their countries of origin, and others may be relapses subsequent to past detection and treatment in either their new or their original countries. The WER table of foreign born cases in 2016, implied that the foreign born cases were newly reported (as they were described as percentages of the newly reported cases), ${ }^{4}$ but this has not been assumed in the reports for 2017-2018. ${ }^{5,6}$ These distinctions become important in trying to ascertain the location where cases were infected. Detailed data on individual patient histories are preferable to tabulations of notified cases for close epidemiological analysis.

Despite such difficulties it has been possible to assemble relevant data from northern Portugal. We find that the data are incomplete, and we have described assumptions which we have used in their interpretation. It is encouraging that the data appear to be more thorough since 2005. Notwithstanding these problems these data suggest a picture of leprosy epidemiology fully consistent with what is known from elsewhere in Europe. Portugal was still endemic for $M$ leprae transmission and disease in the mid-20th century, but the disease declined dramatically over recent decades, and a major current question is whether $M$ leprae transmission continues in northern Portugal at all.

Of the cases which appear to be attributable to infection contracted within Portugal (ie autochthonous), none were born after 1966, and none were notified after 2006. Of the five autochthonous cases notified since 2000, four were clearly relapses, and the other was an individual born in 1920, and thus likely to have been infected long ago if not actually a relapse. Of the 11 cases notified since 2005, three were clear relapses, and eight had evidence of having contracted their infections abroad. Six of these imported cases were from Brazil, which has the second largest number of leprosy cases in the world next to India. Brazil has a particularly close 
relationship to Portugal, with 85,426 Brazilians legally living in Portugal in 2017, accounting for $20 \%$ of foreign residents, by far the largest immigrant community. ${ }^{17}$ The association is thus to be expected.

The data are thus consistent with autochthonous transmission of $M$ leprae having stopped in the five districts of northern Portugal more than 20 - and probably at least 40 - years ago. The fact that there is little evidence of more recent transmission, despite the presence of recent imported cases, is consistent with the experience of other northern European countries. In this context, case number 37 in the series reported here is of particular interest, as it is unknown whether this young girl was infected before or after she came to Portugal with her mother, the likely source of her infection. Of course, given that the incubation period of leprosy may last 20 years or more, ${ }^{18}$ that the onset may be insidious, and that ultimate diagnosis may be delayed for some time after clinical onset, it is possible that a few more autochthonous cases will be recognised, but this is unlikely.

The overall decline in leprosy in Portugal coincides with a period of considerable socioeconomic improvement in the country, whether measured in social, economic or health terms. Though this association has been observed in many countries, its explanation is still not entirely clear - ie the extent to which physical conditions or health behaviours are responsible for continued transmission and clinical manifestation of the infection. In this context we note that a high proportion of NP cases were from rural areas and impoverished backgrounds and several reported close relatives with leprosy. An association with rural populations has been noted in several studies. ${ }^{7,10,18}$ On the one hand this may be considered surprising, insofar as M leprae transmission is supposed to rely on close contact and the respiratory route has been considered important, which one might think would favour crowded urban settings. On the other hand close physical contact is expected anywhere among close relatives in a family and household setting, and there may be something about the living conditions of the rural poor which is particularly conducive to the leprosy bacillus.

We suspect that data of the sort presented here are available in several European countries, and encourage that they be collated and reported to WHO. The fact that WHO has recently (since 2015) begun to collect and publish data on leprosy from European countries should encourage more attention to the subject and lead to an improvement in data quantity and quality. If examined as here, they may add to (or refute) the increasing evidence of the progressive cessation of $M$ leprae transmission in the European Region. Just what are the critical factors responsible for this cessation remain an important question for leprosy research and control worldwide.

\section{Acknowledgements}

We thank: Dr Maria Neto the Regional Health Authority of Northern Portugal for her suggestions and support in the procedures to request data; all Local Health Authorities in Northern Portugal for providing copies of notification forms and other written reports; Dr Judite Catarino, Marina Ramos and Dr Paulo Nogueira from the Epidemiology and Statistics Division of the General Directorate of health, in Lisbon, for providing copies of notification forms; Dr Isabel Santiago Moita for providing useful insights on some of the cases.

\section{Funding}

No funding was received for this study. 


\section{Contributors}

GG designed the study, prepared the protocol which was submitted to Ethical Committee, entered and analysed the data and drafted the paper. IA assisted in design of the study and preparation of the protocol, collected data from municipal health authorities, and made comments on the draft of the paper. CP assisted in design of the study and preparation of the protocol, and made comments on draft paper. PF asked the research question, suggested the study, advised on the protocol and analysis and assisted in drafting the paper. All authors were involved in interpretation of the data and commented on interim drafts. All authors have read and approved the final version of the article. GG is the guarantor.

\section{Declaration of interest}

No conflict of interest to declare.

\section{References}

1 WHO. Handbook of resolutions and decisions of the World Health assembly and the Executive Board, vol III, 1985-1992. 3rd ed., Geneva: WHO 1993; pp. 118-118.

2 Regional Office for Southeast Asia - WHO. Global Leprosy Strategy 2016-2020: Accelerating towards a leprosyfree world. WHO/SEARO 2016.

3 WHO. Global leprosy update, 2015: Time for action, accountability and inclusion. Weekly Epidemiological Record, 2016; 91(35): 405-420.

4 WHO. Global leprosy update, 2016: Accelerating reduction of disease burden. Weekly Epidemiological Record, 2017; 92(35): 501-520.

5 WHO. Global leprosy update, 2017: Reducing the disease burden due to leprosy. Weekly Epidemiological Record, 2018; 93(35): 445-456.

6 WHO. Global leprosy update, 2018: Moving towards a leprosy-free world. Weekly Epidemiological Record, 2019; 94(35/36): 389-412.

7 Irgens LM. Leprosy in Norway: An epidemiological study based on a national patient register. Lepr Rev, 1980; 51(Supplement 1): 1-130.

8 Gill AL, Gill GV, Beeching NJ. Familial transmission of leprosy in post-war Britain-discrimination and dissent. Q J Med, 2008; 101: 407-413.

9 Beek CH. An autochthonous case of leprosy in the Netherlands [in Dutch]. Ned Tijdschr Geneeskd, 1961; 105: 2214-2216.

10 Suárez-Garcia I, Echevarría JRG, Cewrvera FM, Gómez-Barroso D, Fine PEM. The decline of autochthonous leprosy in Valencia Region of Spain: Patterns and trends 1940-2015. Lepr Rev, 2017; 88: 162-173.

11 Leitão AE. Declaração Obrigatória de Doenças Transmissíveis. Saúde em Números, 1998; 13: 17-18.

12 Irgens LM, Melo Caeiro F, Lechat MF. Leprosy in Portugal 1946-80: Epidemiologic patterns observed during declining incidence rates. Lepr Ver, 1990; 61: 32-49.

13 Portugal. Ministério da Saúde. Direção-Geral da Saúde. Despacho no. 5855/2014. Diário da República, 2. série - No. 85 - 5 de maio de 2014, página 11660.

14 Sequeira J, Martins C, Marques C, Machado A, Poiares-Batista A. Hanseníase. Estudo Comparativo entre Doentes Antigos e Recentes. Acta Médica Portuguesa, 2000; 13: 13-17.

15 Medeiros S, Catorze MG, Vieira MR. Hansen's disease in Portugal: Multibacilary patients treated between 1988 and 2003. JEADV, 2009; 23: 29-35.

16 Portugal. Ministério da Saúde. Direção-Geral da Saúde. Lisboa 2017. Doenças de Declaração Obrigatória 2013120164. VOLUME II-Regiões.

17 Portugal. SEF (Serviço de Estrangeiros e Fronteiras) 2017. Relatório de Imigração, Fronteiras e Asilo.

18 Fine PEM. Leprosy: The epidemiology of a slow bacterium. Epidemiologic Reviews, 1982; 4: 162-188. 